\title{
ESTRUTURA DEMOGRÁFICA
}

\section{DA POPULAÇĀO ECONOMICAMENTE ATIVA E DESIGUALDADE DA DISTRIBUIÇĀO DE RENDA*}

\author{
Elenora C. Santos** \\ Simone Wajnman***
}

\section{Resumo}

A preocupação fundamental deste trabalho é tentar quantificar os efeitos das variações na estrutura etária da $\mathrm{PEA}$ por sexo e das variações no perfil de rendimentos médios por idade e sexo sobre a evolução temporal e regional da desigualdade de renda no Brasil, no periodo de 1976 a 1987.

A evolução regional e temporal dos rendimentos médios masculinos e femininos, bem como o diferencial desses rendimentos entre os sexos também constituem objeto da análise.

Palavras-chave: Desigualdade de Renda, População Economicamente Ativa, Estrutura Etária, Perfil de Rendimentos, Diferencial de Rendimentos.

\section{Abstract}

This paper aims at quantifying the effects of the variations in the age structure of the labor force by gender and in the average earnings profile by age and gender. This effects will be quantified under the evolution of income inequality in Brazil, from a regional and temporal approch in the period 1976-1987.

The regional and temporal evolution of female and male average earnings, as well as the gap of these earnings between genders is also analysed.

Key words: Income Inequality, Labor Force, Age Structure, Earnings Profile, Earnings Differential.

\section{Introdução.}

O crescimento da desigualdade ao longo do tempo, assim como os diferenciais regionais da distribuição dos rendimentos no Brasil têm sido objeto de inúmeros estudos. Entretanto, freqüentemente não são considerados os efeitos que estruturas

\footnotetext{
* Es te trabalho foi, em parte, beneficiado pelo Programa Nacional de Pesquisa Eco nômica (PNPE). Gostaríamos de agradecer a Renata, Jerônymo e Jaime Pontes pelo excelente suporte computacional dado à execuçāo desse trabalho. **P esquisadora Visitante do IPEA-RIO.

*** Do CEDEPLAR/UFMG.
}

R. de Econometria Rio de Janeiro v. X, no 2, p.279-311 novembro 1990 
demográficas distintas podem causar sobre as comparações entre os valores associados às medidas de desigualdade. Embora não tenham sido suficientemente explorados na literatura sobre distribuição de renda no Brasil, ${ }^{1}$ esses efeitos têm merecido destaque na literatura internacional, na qual se verifica uma expressiva produção tratando das inter-relações entre desigualdade e estruturas demográficas. ${ }^{2}$

Do ponto de vista da estrutura etária da população, a idéia básica que inspira estes trabalhos é a de que os individuos percebem rendimentos diferenciados ao longo de seus ciclos de vida. Por esta razão, Paglin (1975) observa que há uma parcela da desigualdade que deve ser atribuida às diferenças de rendimento em função da idade, pois a população, em seu conjunto, é formada por indivíduos posicionados em pontos distintos de seus ciclos vitais de rendimentos. Segundo a mesma lógica, há também uma parcela da desigualdade que deve ser atribuída aos diferenciais de rendimentos por sexo, dado que homens e mulheres não são remunerados de forma equivalente. Estas parcelas da desigualdade, portanto, provêm do fato de existirem diferenciais de rendimento relacionados com a idade e o sexo, e explicam uma parte conceitualmente importante da desigualdade global, já que indicam o papel do perfil etário e da discriminação entre os sexos no mercado de trabalho.

Mas, se os rendimentos são uma função, dentre outras coisas, da idade e do sexo dos individuos, a distribuição dos rendimentos é uma função da composição demográfica (estrutura etária e composição por sexo) da população. Assim, a simples mudança na composição etária e por sexo da população acarreta mudanças nas medidas de desigualdade, pois alteram-se as participações relativas dos grupos de idade e sexo que possuem rendimentos médios diferenciados. Este tipo de efeito sobre as medidas de desigualdade, denominado efeito de composição, deve ser removido das comparações entre medidas de desigualdade, pois, embora a capacidade dos individuos de auferir ganhos dependa de sua idade e sexo, o bem-estar da sociedade num dado momento não depende

\footnotetext{
${ }^{1}$ Apenas Langoni (1973), Morley (1979) e Simonsen (1978) procuraram abordar estes efeitos na análise do crescimento da deisgualdade na década de sessenta. Para as décadas de setenta e oitenta, ver, Wajnman (1989) e Ramos (1990).

2 Ver, por xemplo, Paglin (1975), Potter (1979) ou Lam (1984, 1985, 1987).
} 
da composição por idade e sexo da sua população. ${ }^{3}$

Lam (1987), além de explicitar estes efeitos, mostra como a comparação entre distribuições com distintas composições populacionais tende a produzir resultados ambíguos, dificeis não só de serem interpretados, como também de serem previstos. Do ponto de vista dos efeitos de composição associados a estrutura etária, o autor demonstra que o impacto de uma estrutura etária mais jovem sobre a desigualdade global depende de uma combinação de duas componentes. Por um lado, uma estrutura etária mais jovem, ao elevar a participação dos indivíduos que têm rendimentos médios relativamente inferiores na população, tende a elevar a componente "inter-coorte" do total da desigualdade. Por outro lado, se a dispersão "intra-coorte" dos rendimentos tender a se elevar com a idade, o aumento da participação dos jovens na população pode levar a uma redução da componente "intra-coorte" da desigualdade total. O resultado final é que o total da desigualdade depende da combinação das desigualdades intra e intergrupos de idade, que são afetadas diferentemente pela mudança na estrutura etária da população.

Finalmente, é preciso observar que a avaliação do papel das estruturas (e mudanças nas estruturas) demográficas no comportamento da desigualdade assume especial interesse se consideramos que importantes mudanças na estrutura de idade e sexo da População Economicamente Ativa (PEA) estão em curso no Brasil. O envelhecimento da estrutura etária da população, decorrente da marcante queda da fecundidade nas últimas décadas, e o crescimento da participação feminina na força de trabalho vêm marcando o comportamento recente da PEA num processo que ten de a se intensificar.

Assim, o objetivo principal deste trabalho é quantificar os efei tos de duas componentes do comportamento temporal e regional da desigualdade global: (i) as variações na estrutura etárıa da PEA por sexo e, (ii) as variações no perfil de rendimentos méclios por idade e sexo. Chama-se a atenção para o fato de que as variações na primeira componente resultam nos efeitos de composição mencionados, que não têm nenhum impacto sobre o bem-estar da população. 0s efeitos da segunda componente, no

${ }^{3}$ Paglin (1975), Morley (1979) e especialmente Lam (1984, 1985 e 1987) chamar am a atençāo para estes efejtos de composiçāo. 
entanto, constituem parcela importante na explicação da desigualdade. Pretende-se avaliar, também, a evolução dos rendimentos médios de homens e mulheres, para o periodo em análise, dando especial atenção para o diferencial entre ambos. Essa análise, é feita a partir das informações sobre rendimentos, idade e sexo das pessoas economicamente ativas contidas nas PNADS de 1976 a 1987 das nove regiões metropolitanas brasileiras, o que permite que as dimensões temporal e regional do comportamento da desigualdade sejam contempladas no estudo.

\section{Diferenças temporais e regionais na desigualdade.}

Os gráficos 1, 2, 3 e 4 mostram a evolução da desigualdade de rendimentos, medida pelo índice $L$ de Theil, ${ }^{4}$ entre 1976 e 1987, nas nove regiões metropolitanas brasileiras, considerando-se, separadamente, os homens e as mulheres economicamente ativos, de 10 a 70 anos de idade, que contassem com algum rendimento (do trabalho ou não) positivo. ${ }^{5}$ Através dos gráficos 1 e 2 é possível notar que os niveis de desigualdade (desigualdade média e das regiões extremas no que se refere à desigualdade) flutuam ao longo do tempo, sem um padrão muito claro de comportamento, embora, no caso dos homens, se observe mais claramente um perfil na forma de um $\mathrm{U}$ - ou seja níveis mais elevados de desigualdade nos anos extremos ${ }^{6}$ e, no caso das mulheres, haja uma tendência mais definida de crescimento da desigualdade. Os gráficos 3 e 4, que apresentam as médias dos indices $L$ de Theil entre $1976 \mathrm{e}$ 1987 das regiões metropolitanas, mostram como os niveis de desigualdade tendem a ser menores nas regiões localizadas no sudeste

${ }^{4} \mathrm{O}$ indice $L$ de Theil, que é a segunda medida de desigualdade de Theil (Ver Anand, 1983), para uma populaçāo com $n$ indivíduos, onde $X_{3}$ é a renda do i-ésimo indivíduo, pode ser descrito como:

$$
L=\log \bar{X}-\bar{Z}
$$

onde $\bar{X}=1 / n \sum_{i=1}^{n} X_{i}, \bar{Z}-1 / n \sum_{i=1}^{n} Z_{i}$ e $Z_{i}=\log X_{i}$.

Logo, pode-se dizer que o índice $L$ de Theil equivale ao logaritmo da razāo entre as médias aritmética e geométrica das rendas individuais.

${ }^{5} \mathrm{Os}$ dados básicos referentes a estes gráficos encontram-se à disposiçāo, com as autoras.

${ }^{6}$ A mesma observação é feita por Reis e Barros (1989). 
e sul, com São Paulo apresentando os menores indices. Nota-se também que a desigualdade entre as regiões é mais evidente no caso das mulheres.

Observando-se ainda os gráficos 5 e 6 , que comparam os niveis de desigualdade entre homens e mulheres ao longo do tempo (gráfico 5) e através das regiões (gráfico 6), verifica-se, em primeiro lugar, que a desigualdade entre os homens foi maior que entre as mulheres ao longo do periodo analisado, exceto nos anos de 1983, 1984 e 1985. Em segundo lugar, observa-se que, nas regiões onde a desigualdade média é menor, ou seja, as regiões do sudeste e sul, (ver gráficos 3 e 4), os indices de desigualdade para as mulheres são menores que os dos homens.

\section{Padronizaçōes dos índices de desigualdade.}

Nesta seção apresentam-se os resultados dos exercícios de padronização que quantificam os efeitos de composição sugeridos anteriormente. Operacionalmente as padronizações consistem em substituir a estrutura etária efetivamente observada para uma dada região e ano por uma outra adotada como padrão e recalcular o indice de desigualdade que se obtém dessa forma. Em seguida, substitui-se o perfil etário de rendimentos efetivo por um perfil padrão e novamente recalcula-se o indice de desigualdade. As estruturas de idade e rendimentos utilizadas como padrão para comparações regionais foram as de São Paulo, por ser essa a região de menor índice de desigualdade. Para as comparações temporais, adotaram-se as estruturas de 1976 para que os resultados indicassem a evolução temporal da desigualdade na ausência de mudanças na estrutura etária e perfil de rendimentos. A medida de desigualdade padronizada foi o indice $L$ de Theil $(L)$ e chamouse $L_{*}$ o resultado da primeira padronização, ou seja, a medida padronizada pela estrutura etária e, $L * *$ a medida padronizada pelo perfil etário de rendimentos.

1 - O papel da estrutura etária e do perfil etário de rendimentos nas variações regionais da desigualdade

A tabela 1 apresenta uma sintese dos resultados das padronizações dos indices $L$ de Theil relativos às distribuições de rendimentos de homens e de mulheres. ${ }^{7}$ Os resultados dessa tabela

\footnotetext{
${ }^{7}$ Os resultados por regiāo estão à disposiçăo com as autoras.
} 
indicam, em todos os anos, qual seria o nivel médio de desigualdade das regiões $(M)$ e qual seria a dispersão deste nivel entre as mesmas $(C V)$ se, em todas, a PEA tivesse a mesma estrutura etária e o mesmo perfil de rendimentos por idade que os de São Paulo.

Observando-se as médias das primeiras padronizações $\left(M L_{*}\right)$ da tabela 1, que respondem sobre o papel exclusivo da estrutura etária, verifica-se que, no caso dos homens, para todos os anos, adotar a estrutura etária de São Paulo altera, na maioria dos casos diminuindo menos de $1 \%$ a média das desigualdades das regiões. Efeito mais forte e claro tem a utilização do perfil de rendimentos de São Paulo nas médias das padronizações $\left(M L_{* *}\right)$ : em todos os anos este perfil diminui a média das desigualdades das regiões, algo que varia em torno de $4 \%$, diminuindo também o coeficiente de variação entre as regiões $\left(C V_{L}\right)$, em média $23 \%$. Ou seja, se todos os perfis de rendimentos fossem os mesmos e iguais aos de São Paulo, no caso masculino os níveis de desigualdade seriam menores e a dispersão regional da desigualdade seria, em média, $23 \%$ menor.

Quanto às padronizações relativas às mulheres, observa-se que a estrutura etária de São Paulo diminui a desigualdade média em todos os anos, em torno de $3 \% .^{8} 0$ perfil de rendimentos também reduz as médias das desigualdades de todos os anos, em média $10 \%$, e reduz também sempre o coeficiente de variação entre as regiões numa média de $21 \%$ para todos os anos.

Portanto, tanto para homens quanto para mulheres, a estrutura etária e, principalmente, o perfil de rendimentos de São Paulo explicam, em parte, que esta região tenha o menor nivel de desigualdade. Além disso, as diferenças entre as regiões quanto a seus perfis etários de rendimentos explicam parte significativa da dispersão regional da desigualdade.

Esses resultados, contudo, merecem algumas considerações. Do ponto de vista do papel das estruturas etárias de São Paulo, como se observou, embora o efeito não seja de magnitude muito significativa, ele é sistematicamente o mesmo. Ou seja, a estrutura etária da PEA de São Paulo, seja feminina ou masculina, reduz, praticamente sempre, o nivel de desigualdade das demais

${ }^{8}$ Exceção feita para o ano de 1986, quando essa estrutura etária eleva a desjgualdade de todas as regiões. 
regiões. O interessante, no entanto, é observar que isto ocorre por razões inteiramente distintas ıo caso de homens e mulheres. Os gráficos 7 e 8 exemplificam as diferenças de estrutura etária que, via de regra, ocorrem entre São Paulo e as demais regiões. ${ }^{9}$ Como se observa, no caso dos homens (gráfico 7), São Paulo concentra maior proporção de PEA nos grupos de idade onde os niveis de rendimentos são relativamente maiores (ver gráfico 8), enquanto que Fortaleza apresenta um pico de participação da PEA mais à esquerda, ou seja, nas idades para as quais os rendimentos tendem a ser menores. $\mathrm{O}$ impacto disso sobre a diferença entre a desigualdade das duas regiões pode ser de dois tipos: por um lado, ter mais PEA concentrada nos grupos de idade mais alta pode diminuir a componente inter-coorte da desigualdade global de São Paulo, já que estes grupos apresentam rendimentos médios mais elevados. Por outro lado, como a desigualdade dentro de cada grupo etário cresce com a idade, a estrutura etária de São Paulo pode favorecer uma componente intra-coorte da desigualdade mais elevada. Os resultados das padronizações atestam que, nesse caso, o efeito liquido final é uma redução da desigualdade quando adotada a estrutura etária de São Paulo, ou seja, prevalece o efeito de um rebai xamento da desigualdade inter-coorte. No caso das mulheres, ao contrário, como se exemplifica no gráfico 9, a PEA de São Paulo apresenta maior concentração de mulheres mais jovens que as demais regiões. Novamente, isto representa dois tipos de efeitos, de sinais contrários, sobre a desigualdade. Neste caso, ao contrário dos homens, prevalece o efeito de uma redução na componente intra-coorte da desigualdade global, já que, como se viu, adotar como padrão a estrutura etária de São Paulo significa diminuir a desigualdade das demais regiões.

\section{O papel da estrutura etária e do perfil etário de ren- dimentos na evolução temporal da desigualdade.}

A tabela 2 apresenta as padronizações dos indices de desigualdade, na dimensão temporal, onde se utilizou como padrão as estru turas de 1976. Com esses resultados é possivel avaliar qual seria a evolução temporal da desigualdade, em cada uma das regiões

\footnotetext{
${ }^{9}$ Nesses gráficos aparecem apenas as estruturas de Sāo Paulo, que apresentam desigualdade mínima, e da regiāo de máxima desigualdade.
} 
metropolitanas, caso não tivesse havido mudança nas estruturas de idade e rendimentos entre 1976 e 1987.

Numa abordagem bastante geral, ou seja, considerando-se conjuntamente todas as regiões, não se encontra muita regularidade no resultado das padronizações. No caso dos homens, pelos resultados das médias das primeiras padronizações $\left(M L_{*}\right)$, podese afirmar que a estrutura etária de 1976 eleva a desigualdade média de Belém, Fortaleza, Belo Horizonte, Curitiba e Porto Alegre, além da de São Paulo, mas, em média, menos de $1 \%$, indicando apenas que, ao longo do tempo, nessas regiões, a mudança na estrutura etária contribuiu para a redução da desigualdade. Nas demais regiões (Recife, Salvador e Rio), a mudança na estrutura etária da PEA atuou no sentido contrário, impedindo uma maior queda dos níveis de desigualdade.

Os resultados médios da segunda padronização $\left(M L_{* *}\right)$ mostram que, com exceção de Belo Horizonte, Rio de Janeiro e Porto Ale je, o perfil etário de rendimentos de 1976 levou a maiores indices da desigualdade média, no caso masculino, ou seja, nestas regiões, a mudança efetivamente ocorrida no perfil etário de rendimentos também contribuiu para a diminuição da desigualdade. Observa-se, finalmente, que $26 \%$, em média, da variabilidade ao longo dos anos deve-se às mudanças ocorridas nos perfis de rendimentos. ${ }^{10}$

Consideranto-se todas as regiões na tabela 3 , no caso das mulheres constata-se que a distribuição etária de 1976 reduz todas as desigualdades, com exceção da de Curitiba, em média $3 \%$. Assim, a mudança da estrutura etária que efetivamente ocorreu contribuiu para o aumento da desigualdade média de todas as regiões, menos Curitiba. Possivelmente, esse aumento se deu com o envelhecimento da estrutura etária da PEA, via elevação da componente intra-coorte. Já a mudança no perfil de rendimentos não produziu resultados muito regulares através das regiões. $O$ perfil de 1976 reduz a desigualdade média de Belém, Fortaleza, Belo Horizonte, Rio de Janeiro e São Paulo, mantém constante a de Salvador e aumenta a das demais regiões. Verificando-se a alteração do coeficiente de variação com a padronizaç̧ão, conclui-se que, para as mulheres, em média, $33 \%$ da variabilidade dos índices

${ }^{10} \mathrm{O}$ coeficiente de variaçāo cai, em média $26 \%$ quando se padroniza o indice $L$ de Theil pelo perfil etário de rendimentos de 1976. 
$L$ de Theil ao longo do tempo deve-se a mudanças no perfil etário de rendimentos.

5. O papel da estrutura etária e do perfil etário de rendimentos nos diferenciais de desigualdade entre homens e mulheres.

Por fim, nas tabelas 3 e 4 apresentam-se as padronizaçōes que avaliam o impacto das diferenças existentes entre as estruturas etárias e os perfis de rendimentos femininos e masculinos nos indices $L$ de Theil de homens e de mulheres. Nesse caso, optou-se, arbitrariamente, por apresentar apenas as padronizaçōes do ano de 1985, para a comparação entre as regiōes, e as de São Paulo para a análise temporal, $\mathrm{j}$ a que a descrição das demais seria muito extensa.

As padronizaçōes para 1985, constantes na tabela 3, mostram o efeito liquido final das diferenças entre as estruturas femininas e masculinas. No caso específico de São Paulo, ilustrado nos gráficos 11 e 12, a padronização que associa a estrutura etária da PEA feminina ao indice de desigualdade masculino $\left(L_{*}\right)$, gera um indice menor que o original masculino. Ou seja, a estrutura etária da PEA feminina favorece uma menor desigualdade que a estrutura etária dos homens. Além disso, o perfil de rendimentos femininos, conforme indica a segunda padronização $\left(L_{* *}\right)$, também favorece un Paulo, a diferença entre as desigualdades de homens e mulheres é, em parte (42\%), explicada pela diferença entre as estruturas etárias, mas é a diferença entre os perfis de rendimentos que expli ca o fato da desigualdade dos homens ser maior.

Observando-se os outros resultados da tabela 3, verifica-se qua, exceto no caso de Fortaleza, a estrutura etária feminina sempre favorece menor desigualdade como efeito líquido final e, em todos os casos, o perfil de rendimentos femininos também gera menor indice de desigualdade. Assim, comparando-se as diferenças observadas entre os índices $L$ de Theil femininos e masculinos efetiv amente observados com as diferenças entre os indices femininos e masculinos expurgados das diferenças entre estruturas de idade e r endimentos, conclui-se que: (i) nos casos onde a desigualdade masculina é maior que a feminina (São Paulo, Belo Horizonte, $\mathrm{Cu}$ riti ba e Porto Alegre) isto se dá por causa das diferenças de estrutur as de idade e rendimentos, e (ii) nos casos onde, ao contrário 
a desigualdade entre as mulheres é maior que entre os homens (demais regiões), isto se dá apesar das diferenças nas estruturas etária e de rendimento, pois caso as estruturas fossem iguais as diferenças seriam ainda maiores.

A tabela 4 repete o exercício, analisando São Paulo na dimensão temporal. Por esses resultados, observa-se que, no caso específico de São Paulo, a desigualdade masculina é sempre maior que a feminina. Nota-se também que a estrutura etária feminina reduz a desigualdade masculina nos anos de 1976, 1977, 1981, 1985 e 1987 . Em 1984 e 1986 a desigualdade masculina fica elevada quando padronizada pela estrutura etária feminina, e nos outros anos (1978, 1979, 1982 e 1983) fica inalterada, não havendo, dessa forma, um padrão claro de comportamento. Por outro lado, o perfil etário de rendimentos femininos, novamente, sempre diminui a desigualdade masculina. Nos anos de 1977, 1981, 1982, 1983, 1984, 1985 e 1987 essa redução torna a desigualdade entre os homens inferior àquela entre as mulheres. Assim, nestes anos a desigualdade masculina só é maior que a feminina em função das diferenças entre os perfis etários de rendimentos. Nos outros anos, os homens apresentariam maiores indices de desigualdade mesmo se possuíssem as estruturas femininas, mas o diferencial de indices entre homens e mulheres ficaria reduzido, em média, em $88 \%$.

\section{Os diferenciais de rendimentos entre os sexos.}

Até aqui, considerou-se apenas as diferenças entre indices de desigualdade de homens e mulheres, não tendo sido abordadas, entretanto, as diferenças entre os rendimentos médios de homens e mulheres, ou seja, a frópria desigualdade de rendimentos entre os sexos, que também explica parte da desigualdade global. Embora a questão da diferenciação de rendimentos requeira um exame mais detalhado, que inclua variáveis de atributos individuais, tais como idade, número de anos de estudo e experiência no mercado de trabalho, as quais qualificam os diferenciais entre os sexos, tal análise excede as pretensões desse trabalho. Essa seção limita-se apenas a descrever a evolução temporal e regional dos rendimentos médios femininos comparados aos masculinos. ${ }^{11}$

"Santos (1989), analisa o diferencial de rendimentos entre homens e mulheres, dentro de determinados grupos e sub-grupos ocupacionais investigados pelo Censo Demográfico de 1980. Como resultado, encontra que, o diferencial de 
Do ponto de vista das diferenças regionais constata-se que, as regiões estão posicionadas segundo os rendimentos médios ${ }^{12}$ tanto de homens quanto de mulheres, na mesma ordem crescente, a saber: Fortaleza, Recife, Belém, Belo Horizonte, Salvador, Curitiba, Rio de Janeiro, Porto Alegre, São Paulo. Assim, de um modo geral, as regiões que apresentam maiores rendimentos médios são também aquelas onde, como se observou anteriormente, existem os menores níveis de desigualdade entre os rendimentos, sendo a região de São Paulo, a que apresenta os maiores rendimentos médios femininos e masculinos e os menores índices $L$ de Theil.

Do ponto de vista da evolução tempoxral dos rendimentos médios, verifica-se que, também há uma tendência similar no comportamento dos rendimentos masculinos e femininos. Ou seja, esses rendimentos tendem a decrescer no final dos anos setenta e início dos oitenta, apresentando alguma recuperação a partir de 1983. Note-se, entretanto, que desconsiderando-se o atípico ano de 1986, a tendência geral é de queda nos rendimentos, mais acentuadamente no caso masculino. Analisando-se ainda a evolução dos coeficientes de variação, constata-se que a tendência geral é de uma redução na dispersão relativa dos rendimentos médios entre as regiōes, ao longo do tempo.

Finalmente, é preciso chamar a atenção para o fato de que os ren dimentos médios masculinos são muito superiores aos femininos em todos os anos e regiões. Entretanto, observa-se que, ao longo do tempo a diferença entre esses rendimentos tende a decrescer, o que pode ser comprovado através dos resultados constantes na tabela 5 .

A tabela 5 e o gráfico 13 apresentam os rendimentos médios relativos, que são a razão entre, os rendimentos femininos e os masculinos. Nesse sentido, Belo Horizonte caracteriza-se como a reg ião mais "discriminadora" (onde esta razão é mais próxima de zerv) e Recife como o caso contrario. Chama a atenção o fato de

renstimentos entre homens e mulheres, em $90 \%$ das ocupações, apresenta-se favorável ao sexo masculino, e que de maneira genérica, os estados do Sul e Sud este apresentam menores diferenciais quando comparados aos do Norte e Nor deste.

${ }^{12} \mathrm{O}$ s rendimentos médios calculados foram reduzidos a salários múnimos deflacrionados, adotando-se como deflator o índice de Freços ao Consumidor (IP disposição com as autoras. 
que a ordenação das regiões segundo os rendimentos relativos não se relaciona com a escala das regiões segundo as desigualdades internas de homens e mulheres. Ou seja, as regiões onde há mais discriminação não são aquelas onde há maior desigualdade. Da mesma forma, nas regiões do pais onde os rendimentos femininos e masculinos são os mais elevados, o diferencial entre eles não é, de maneira genérica, menor.

Passando-se para uma análise temporal, pode-se dizer que, de maneira geral, o diferencial de rendimentos entre os sexos tem diminuido ao longo do periodo compreendido entre 1976 e 1986. O gráfico 18 ilustra essa tendência, através da curva da média dos rendimentos relativos, e apresenta as duas regiões, Belo Horizonte e Recife, onde esse diferencial corresponde, respectivamente, aos extremos menor e maior dessa relação. Embora, como já dito, a tendência, tanto nessas duas regiões quanto nas demais, seja de redução do diferencial entre os sexos (crescimento do rendimento médio relativo), não se observa um padrão muito bem delineado.

\section{Conclusão.}

Dos resultados obtidos com os exercícios de padronização apresentados anteriormente, alguns merecem ser sumarizados e enfatizados à guisa de conclusão. Em primeiro lugar, constatouse, que, via de regra, a desigualdade global é muito mais sensivel a variações no perfil etário de rendimentos do que na estrutura etária da PEA. Especificamente, nas comparações regionais entre indices de desigualdade, constatou-se que, na maioria dos casos, a estrutura etária masculina de São Paulo (mais envelhecida) diminui a desigualdade da regiões, embora esse efeito seja de pequena magnitude. O perfil de rendimentos de São Paulo, menos alongado, diminui também a desigualdade de todas as regiões, além de diminuir a desigualdade entre as regiões. No caso das mulheres, a estrutura etária de São Paulo reduz também a desigualdade das demais regiões, mas, inversamente ao que ocorre com os homens, por ser mais uma estrutrura etária mais jovem. O perfil de rendimentos feminino de São Paulo também reduz as desigualdades das regiões (em média mais do que o perfil masculino o faz) e entre as regiões. Assim, do ponto de vista da dispersão regional, os efeitos captados parecem ser de que a dispersão regional da desigualdade pode ser explicada algo em torno de $20 \%$ pelas dife- 
renças nos perfis etários de rendimentos e, que as diferenças nas estruturas etárias explicam muito pouco da dispersão regional.

$\mathrm{Na}$ dimensão temporal, concluiu-se, por um lado, que as mudanças na estrutura etária da PEA masculina ao longo do tempo contribuiram, na maioria das regiões, para reduzir a desigualdade, embora este efeito tenha sido discreto. Por outro lado, no caso das mulheres, as mudanças na estrutura etária contribuíram para a elevação da desigualdade, sendo esse efeito mais forte que no caso dos homens. As mudanças nos perfis de rendimentos médios efetivamente observadas através dos anos, no caso dos homens, favoreceram a redução da desigualdade e, pode-se também afirmar que, em média, $26 \%$ da variabilidade ocorrida ao longo do tempo, deve-se a essas mudanças. No caso das mulheres, o impacto das mudanças de perfil de rendimentos não definem um padrão claro, já que para algumas regiões significou redução, para outras aumento e, para outras ainda não teve nenhum papel na evolução da desigualdade.

Quanto ao papel das estruturas de idade e rendimentos sobre o diferencial de desigualdade entre os sexos, constatou-se que, na maioria dos casos, a estrutura etária, assim como o perfil de rendimentos femininos, favorecem um menor nivel de desigualdade, como efeito final. Em alguns casos (São Paulo, Belo Horizonte, Curitiba e Porto Alegre) são essas diferenças na estrutura etária e perfil de rendimentos que explicam que a desigualdade entre os hom $e$ ns seja maior que entre as mulheres. Nos outros casos, apesar dessas estruturas favorecerem uma maior desigualdade entre os homens, isto não é o suficiente para impedir que a desigualdade entre as mulheres seja maior, dado que estas apresentam uma estrutura de desigualdades dentro dos grupos etários, ou seja, uma desigualdade não explicada pela idade, muito maior que a dos homens.

Finalmente, no que tange às diferenças de rendimentos entre os sexos, pode-se constatar que, embora os rendimentos médios masculinos sejam sistematicamente maiores que os femininos, este dife rencial decresceu ao longo dos anos analisados, em todas as regi ões, o que significa que a desigualdade entre os sexos perde import ância para explicar a desigualdade global. Verificou-se ainda que, não necessariamente onde ocorreram menores desigualdades entre os rendimentos, tanto masculinos quanto femininos, os dife- 
renciais de rendimentos entre os sexos foram, via de regra, menores. Além disso, as regiões do Sul e Sudeste, nas quais os rendimentos médios femininos e masculinos são os maiores, são exatamente aquelas em que há menores niveis de desigualdade de renda.

\section{Referências}

IBGE (Instituto Brasileiro de Geografia e Estatistica). Pesquisa Nacional por Amostra de Domicilios (PNAD). Rio de Janeiro, IBGE, 1976, 1977, 1978, 1979, 1981, 1982, 1983, 1984, 1985,1986 e 1987.

Lam, D. The variance of population characteristics in stable populations, with applications to the distribution of income. Population Studies, 38:117-27, 1984.

The dynamics of population growth, differential fertility, and inequality. University of Michigan, research reports, $\mathrm{n}$ 85-69.

Distribution issues in the relationship between population growth and economic development. In Johnson, D. G. \& Lee, R. D. population growth \& economic development: issues and evidence. The University of Wisconsin Press. 1987. Langoni, C.G. Distribuição de Renda e Desenvolvimento Econômico no Brasil. Rio de Janeiro, Editora Expressão e Cultura, 1973.

Morley, S.A. O efeito do crescimento demográfico sobre medidas de distribuição da renda. Pesquisa e Planejamento Econômico, 9(3): 559-592, 1979.

Paglin, M. The measurement and trend of inequality: a basic revision. American Economic Review, 65(3): 520-531, 1975.

Potter, J.E. Demographic factors and income distribution in Latin America. In International union for the scientific study of population. Economic and Demographic Change: Issues for the 1980's. Helsinski. 1979, pp. 321-336.

Ramos, L.R.A. The distribution of earnings in Brazil: 1976-1985. Ph.D. dissertation. Berkeley, University of California. 1990.

Reis, J.G.A. \& Barros, R.P. Income Inequality and the Distribution of Education: A Study of the Evolution of Brazilian Regional Differences in Inequality. Yale University, Micro-Economics Workshop in Labor and Population. 
Santos, E.C. O mercado de trabalho: uma análise do diferencial de remuneração - Brasil 1980. UFMG. Monografia de graduação. 1989.

Simonsen, M.H. Desigualdade e mobilidade social. In Suplicy et. al. debate: economistas contestam Simonsen. Ensaios de Opinião. 1978.

Wajnman, S. Estruíura demográfica da população ecunomicamente ativa e distribuição de renda: Brasil - 1.970/1980. Belo Horizonte, CEDEPLAR/UFMG. (dissertação de mestrado em demografia). 
Tabela 1.

Índices $L$ de Theil e Índices Padronizados pela Estrutura etária e perfil de rendimentos de São Paulo

\begin{tabular}{lllllll}
\hline Anos & $M L$ & $M L^{*}$ & $M L^{* *}$ & $C V L$ & $C V L^{*}$ & $C V L^{* *}$ \\
\hline \multicolumn{7}{c}{ Homens } \\
\hline 1976 & 0.603 & 0.605 & 0.578 & 0.112 & 0.122 & 0.090 \\
1977 & 0.545 & 0.542 & 0.526 & 0.133 & 0.130 & 0.090 \\
1978 & 0.532 & 0.532 & 0.521 & 0.094 & 0.087 & 0.073 \\
1979 & 0.527 & 0.525 & 0.505 & 0.092 & 0.092 & 0.069 \\
1981 & 0.498 & 0.496 & 0.484 & 0.209 & 0.206 & 0.089 \\
1982 & 0.510 & 0.507 & 0.488 & 0.099 & 0.097 & 0.077 \\
1983 & 0.538 & 0.534 & 0.512 & 0.111 & 0.112 & 0.089 \\
1984 & 0.552 & 0.548 & 0.533 & 0.079 & 0.080 & 0.060 \\
1085 & 0.594 & 0.595 & 0.562 & 0.088 & 0.088 & 0.069 \\
1086 & 0.572 & 0.570 & 0.552 & 0.090 & 0.089 & 0.066 \\
1087 & 0.592 & 0.588 & 0.544 & 0.123 & 0.122 & 0.099 \\
& & & & & & \\
\hline & & & & & \\
1976 & 0.532 & 0.518 & 0.454 & 0.286 & 0.266 & 0.193 \\
1977 & 0.518 & 0.500 & 0.435 & 0.385 & 0.375 & 0.312 \\
1978 & 0.472 & 0.463 & 0.439 & 0.193 & 0.192 & 0.153 \\
1979 & 0.466 & 0.452 & 0.417 & 0.213 & 0.211 & 0.171 \\
1981 & 0.483 & 0.470 & 0.445 & 0.267 & 0.266 & 0.134 \\
1982 & 0.507 & 0.494 & 0.462 & 0.288 & 0.286 & 0.157 \\
1083 & 0.547 & 0.531 & 0.465 & 0.184 & 0.175 & 0.143 \\
1084 & 0.593 & 0.577 & 0.543 & 0.181 & 0.178 & 0.146 \\
1985 & 0.607 & 0.595 & 0.560 & 0.174 & 0.170 & 0.145 \\
1986 & 0.539 & 0.555 & 0.505 & 0.181 & 0.177 & 0.171 \\
1987 & 0.565 & 0.550 & 0.514 & 0.202 & 0.204 & 0.171 \\
\hline
\end{tabular}

$M L, M L^{*}, M L^{* *}$ - Média dos Índices $L$ de Theil original, padronizado pela estrutura etária de Sào Paulo c, padronizado pela estrutura etária e perfil de rendimentos de Sào Paulo, respectivamente.

$C V L, C V L^{*}, C V L^{*}$ - Coeficiente de Variação dos Índice $L$ de Theil original, padronizado pela estrutura etária de de São Paulo e, padronizado pela estrutura etária e perfil de rendimentos de Sào Paulo, respectivamente. 


\section{Tabela 2.}

Índices $L$ de Theil $\epsilon$ Índices Padronizados pela Estrutura Etária e perfil de Rendimentos de São Paulo

\begin{tabular}{lcccccc}
\hline & $M L$ & $M L^{*}$ & $M L^{* *}$ & CVL & CVL* & CVL ** \\
\hline Belém & 0.579 & 0.582 & 0.616 & 0.111 & 0.122 & 0.073 \\
Fortaleza & 0.610 & 0.617 & 0.616 & 0.047 & 0.130 & 0.034 \\
Recife & 0.586 & 0.575 & 0.593 & 0.079 & 0.056 & 0.056 \\
Salvador & 0.586 & 0.577 & 0.598 & 0.118 & 0.080 & 0.080 \\
Belo Horizonte & 0.579 & 0.581 & 0.566 & 0.082 & 0.057 & 0.057 \\
Rio de Janeiro & 0.555 & 0.548 & 0.531 & 0.066 & 0.066 & 0.066 \\
Sāo Paulo & 0.456 & 0.460 & 0.460 & 0.058 & 0.044 & 0.044 \\
Curitiba & 0.512 & 0.514 & 0.532 & 0.081 & 0.056 & 0.056 \\
Sāo Paulo & 0.498 & 0.502 & 0.489 & 0.063 & 0.060 & 0.060 \\
& & & & & & \\
\hline & & & Mulheres & & & \\
Belém & 0.611 & 0.595 & 0.589 & 0.228 & 0.203 & 0.159 \\
Fortaleza & 0.617 & 0.598 & 0.584 & 0.125 & 0.126 & 0.095 \\
Recife & 0.655 & 0.638 & 0.767 & 0.155 & 0.166 & 0.085 \\
Sal vador & 0.598 & 0.573 & 0.598 & 0.318 & 0.135 & 0.093 \\
Belo Horizonte & 0.525 & 0.507 & 0.469 & 0.139 & 0.136 & 0.087 \\
Rio de Janeiro & 0.544 & 0.525 & 0.536 & 0.113 & 0.095 & 0.077 \\
Sāo Paulo & 0.384 & 0.371 & 0.369 & 0.098 & 0.090 & 0.081 \\
Curitiba & 0.407 & 0.413 & 0.423 & 0.104 & 0.109 & 0.076 \\
Sāo Paulo & 0.429 & 0.404 & 0.437 & 0.187 & 0.163 & 0.097 \\
\hline
\end{tabular}

$M L, M L^{*}, M L^{* *}-$ Média dos Índices $L$ de Theil original, padronizado pela estrutura etária de 1976, padronizado pela estrutura etária e perfil de rendimentos de 1976, respectivamente.

$C V L, C V L^{*}, C V L^{* *}$ - Coeficiente de Variaçāo dos Índices $L$ de Theil original, Dadronizado pela estrutura etária de 1976 e, padronizado pela estrutura etári a e perfil de rendimentos de 1976, respectivamente. 
Tabela 3.

Índices $L$ de Theil e Índices Padronizados pela Estrutura Etária e Perfil de Rendimentos Femininos - 1985

\begin{tabular}{lcccc}
\hline & $L(\mathrm{fem})$ & $L$ (masc) & $M L^{*}$ & $L^{* *}$ \\
\hline Belém & 0.626 & 0.599 & 0.597 & 0.527 \\
Fortaleza & 0.718 & 0.656 & 0.661 & 0.631 \\
Recife & 0.768 & 0.630 & 0.623 & 0.571 \\
Salvador & 0.706 & 0.638 & 0.631 & 0.574 \\
Belo Horizonte & 0.588 & 0.522 & 0.597 & 0.527 \\
Rio de Janeiro & 0.619 & 0.608 & 0.604 & 0.539 \\
São Paulo & 0.460 & 0.479 & 0.471 & 0.403 \\
Curitiba & 0.506 & 0.555 & 0.543 & 0.477 \\
São Paulo & 0.469 & 0.558 & 0.553 & 0.472 \\
\hline
\end{tabular}

Obs.: $L^{*}-L$ de Theil original masculino padronizado pela estrutura etária feminina da respectiva regiāo em 1985.

$L^{* *}$ - $L$ de Theil masculino padronizado pela estrutura etária e perfil de rendimentos femininos da respectiva regiāo em 1985.

Tabela 4.

Índices $L$ de Theil e Índices Padronizados pela Estrutura Etária e Perfil de Rendimentos Femininos - São Paulo

\begin{tabular}{ccccc}
\hline & $L(\mathrm{fem})$ & $L(\mathrm{masc})$ & $L^{*}$ & $L^{* *}$ \\
\hline 1976 & 0.364 & 0.490 & 0.481 & 0.381 \\
1977 & 0.380 & 0.463 & 0.461 & 0.336 \\
1978 & 0.360 & 0.465 & 0.465 & 0.365 \\
1979 & 0.358 & 0.444 & 0.444 & 0.350 \\
1981 & 0.367 & 0.422 & 0.419 & 0.343 \\
1982 & 0.358 & 0.413 & 0.435 & 0.333 \\
1983 & 0.357 & 0.435 & 0.476 & 0.350 \\
1984 & 0.449 & 0.474 & 0.543 & 0.399 \\
1085 & 0.460 & 0.479 & 0.471 & 0.403 \\
1086 & 0.411 & 0.494 & 0.505 & 0.427 \\
1087 & 0.381 & 0.435 & $0.428^{-}$ & 0.372 \\
\hline
\end{tabular}

Obs.: $L^{*}-L$ de Theil masculino padronizado pela estrutura etária feminina de Sāo Paulo no respectivo ano.

$L^{* *}-L$ de Theil masculino padronizado pela estrutura etária de e perfil de rendimentos femininos de São Paulo no respectivo ano. 
Tabela 5.

Rendimento Médio Relativo Sgundo os Anos em Estudo por REgião Metropolitana - 1976 a 1986 ( $\left.^{*}\right)$

\begin{tabular}{lcccccccccc}
\hline Anos & Bel & For & Rec & Sal & BHt & Rio & SPa & Cur & PoA & média \\
\hline 1976 & 0.37 & 0.43 & 0.57 & 0.35 & 0.33 & 0.48 & 0.45 & 0.37 & 0.49 & 0.44 \\
1977 & 0.59 & 0.39 & 0.42 & 0.43 & 0.38 & 0.49 & 0.47 & 0.45 & 0.45 & 0.45 \\
1978 & 0.44 & 0.44 & 0.43 & 0.41 & 0.45 & 0.47 & 0.48 & 0.43 & 0.46 & 0.45 \\
1979 & 0.42 & 0.46 & 0.52 & 0.44 & 0.44 & 0.51 & 0.48 & 0.44 & 0.46 & 0.47 \\
1981 & 0.53 & 0.47 & 0.51 & 0.44 & 0.46 & 0.52 & 0.51 & 0.52 & .050 & 0.49 \\
1982 & 0.49 & 0.49 & 0.49 & 0.47 & 0.49 & 0.50 & 0.51 & 0.50 & 0.51 & 0.50 \\
1983 & 0.50 & 0.48 & 0.53 & 0.45 & 0.45 & 0.51 & 0.49 & 0.51 & 0.53 & 0.49 \\
1984 & 0.52 & 0.49 & 0.56 & 0.50 & 0.49 & 0.54 & 0.51 & 0.53 & 0.51 & \\
1085 & 0.54 & 0.50 & 0.54 & 0.51 & 0.45 & 0.52 & 0.54 & 0.50 & 0.50 & \\
1086 & 0.53 & 0.49 & 0.61 & 0.54 & 0.48 & 0.55 & 0.51 & 0.49 & 0.52 & 0.52 \\
& & & & & & & & & & \\
Média & 0.49 & 0.48 & 0.51 & 0.47 & 0.45 & 0.50 & 0.49 & 0.49 & 0.50 & - \\
\hline
\end{tabular}

(*) Razão entre o rendimento médio feminino e o masculino.

Fonte: IBGE, PNADs de 1976 a 1986. 

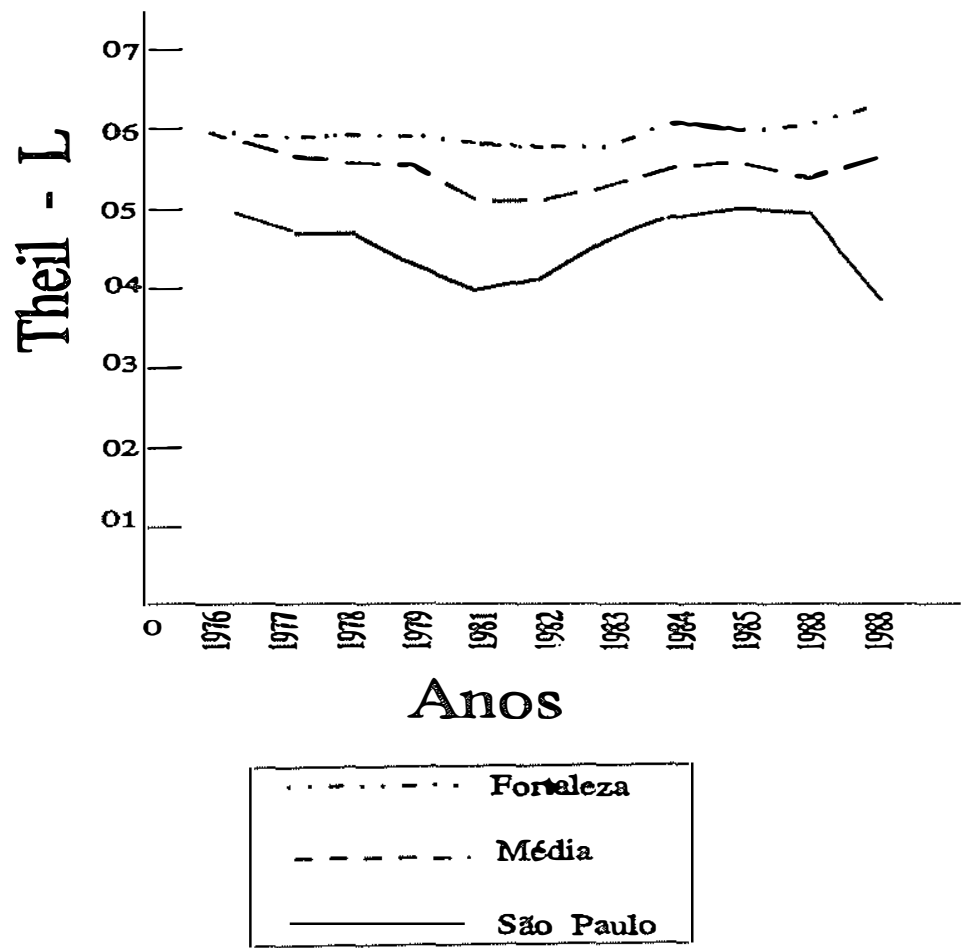

Figura 1.

Evolução da desigualdade de renda.

Regiōes metropolitanas - mulheres 


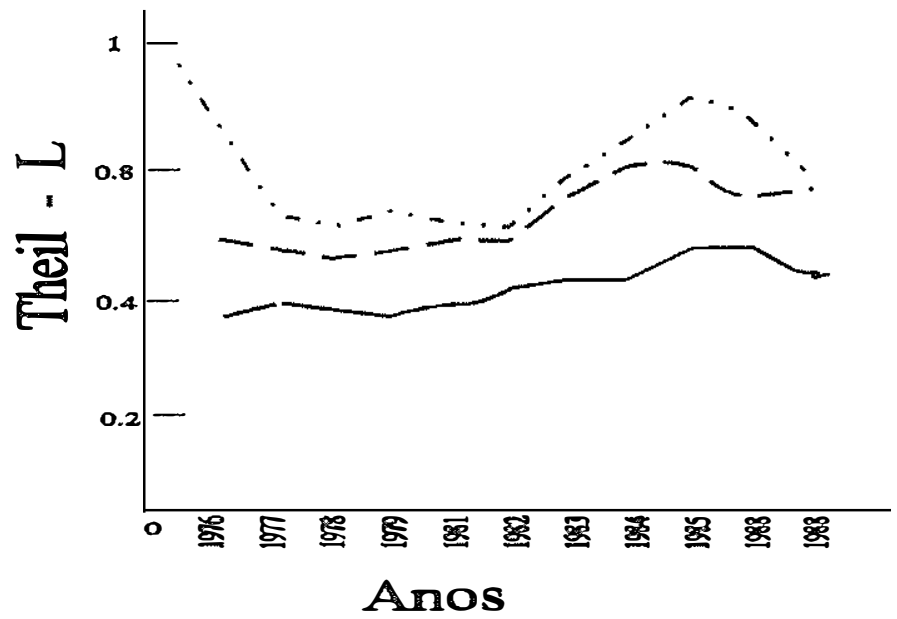

$$
\begin{aligned}
& \cdots-\cdots \cdot \text { Rocife } \\
& -\ldots \text { Média } \\
& \text { Săo Paulo }
\end{aligned}
$$

Figura 2.

Evolução da desigualdade de renda.

Regiōes metropolitanas - homens 


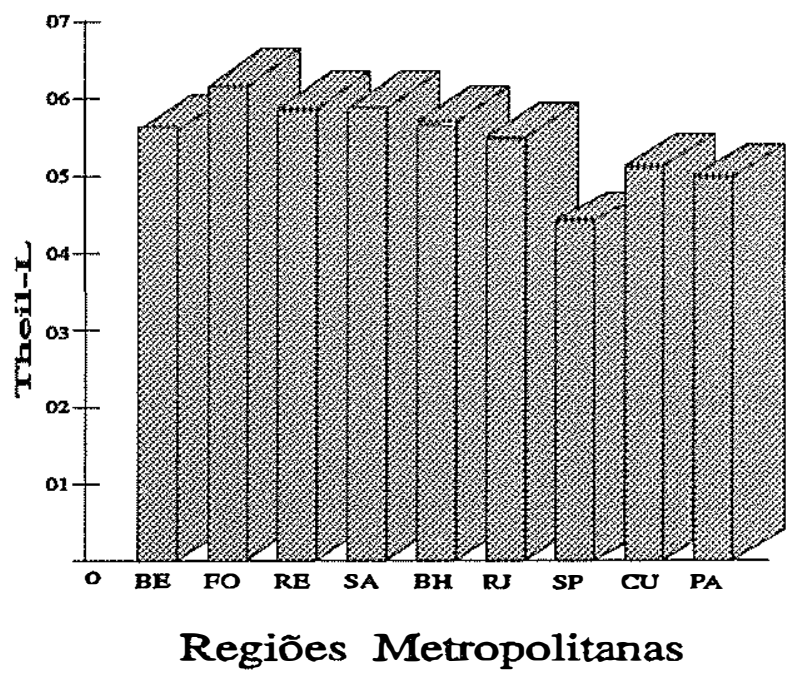

Figura 3.

Desigualdade de renda por regiōes metropolitanas - média $76 / 87$ homens 


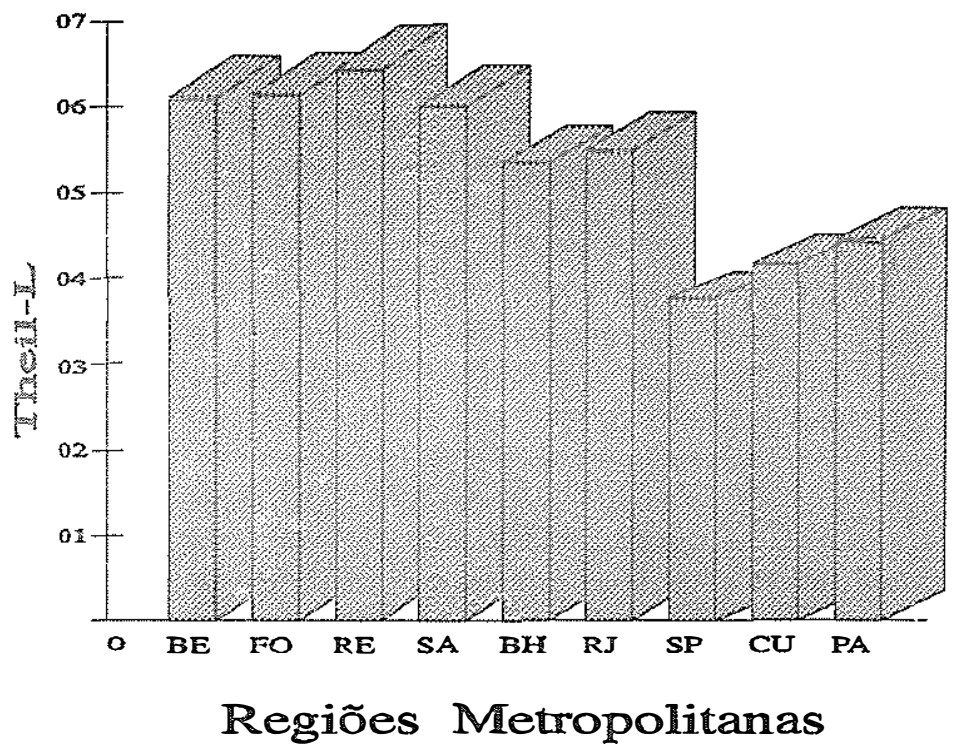

Figura 4.

Desigualdade de renda por regiōes metropolitanas - 76/87 mulheres 

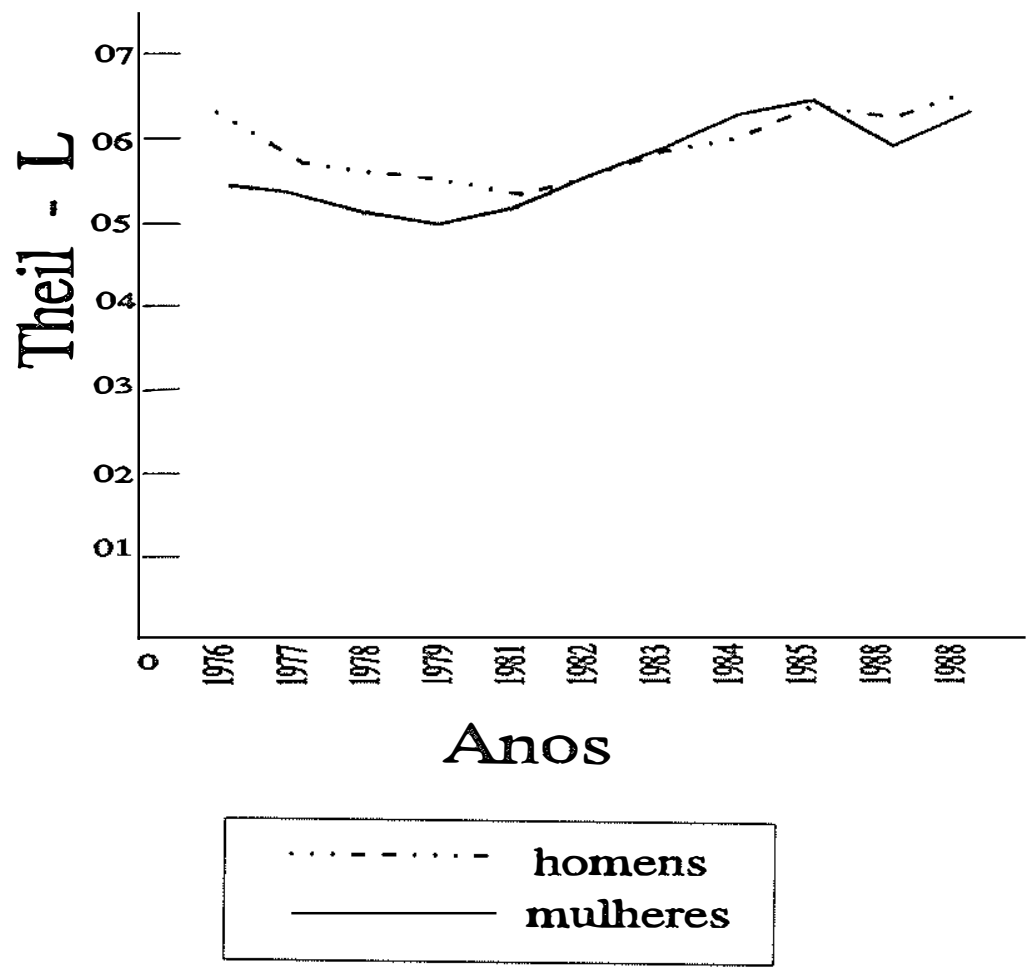

Figura 5.

Evolução da desigualdade de renda.

Homens e mulheres - médias das regōes 


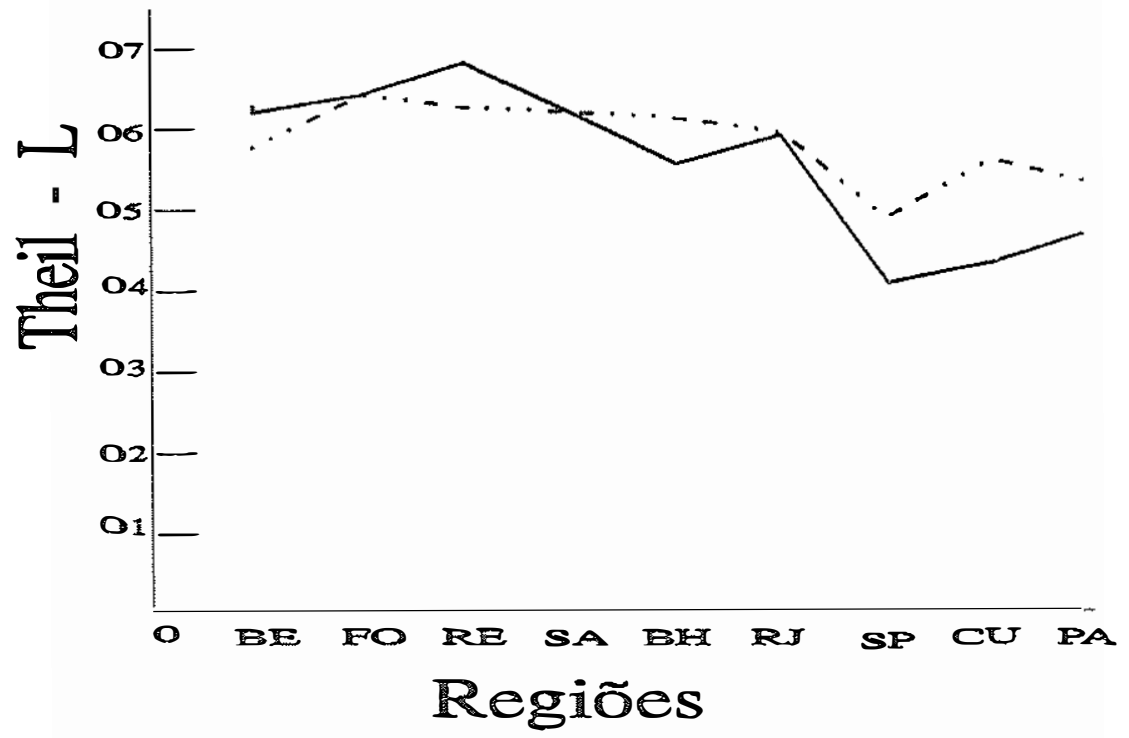

$$
\begin{array}{ll}
\cdots \ldots-\cdots & \text { homens } \\
\text { mulheres }
\end{array}
$$

Figura 6.

Evolução da desigualdade de renda.

Homens e mulheres - médias das regōes 76/87 


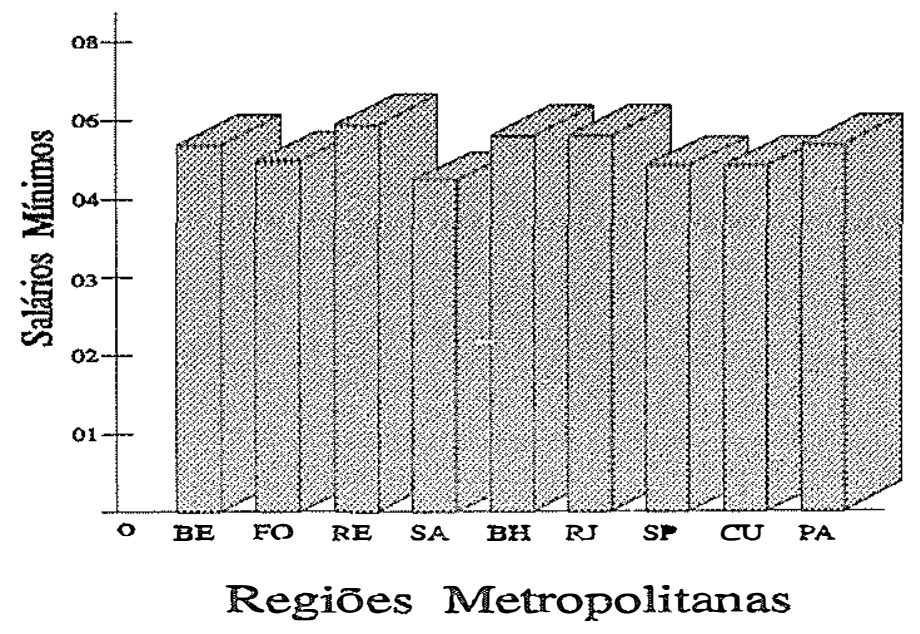

Figura 7.

Distribuição etária da PEA

Homens - 1985 

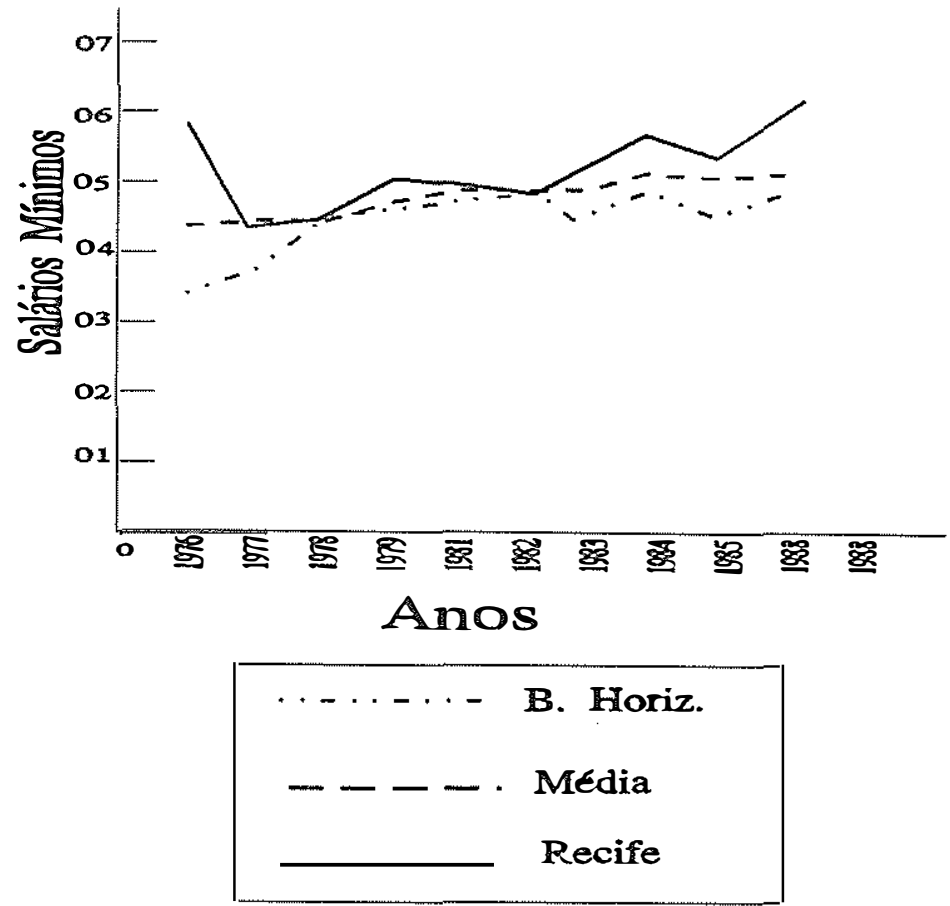

Figura 8.

Perfil etário de rendimentos médios

Masculinos - a PEA 1985 

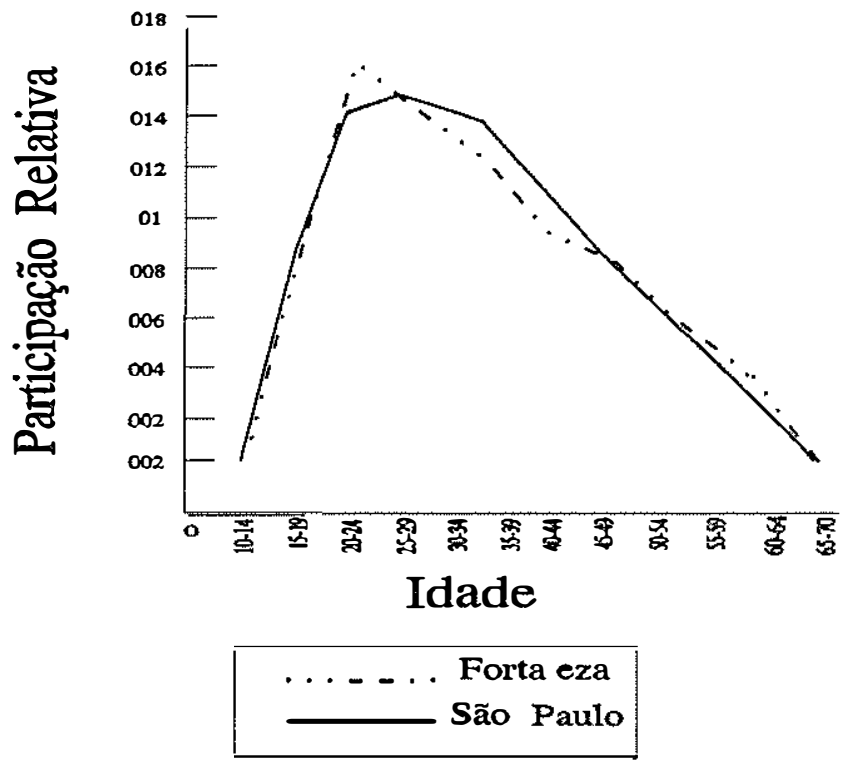

Figura 9.

Distribuição etária da PEA

Mulheres - 1985 


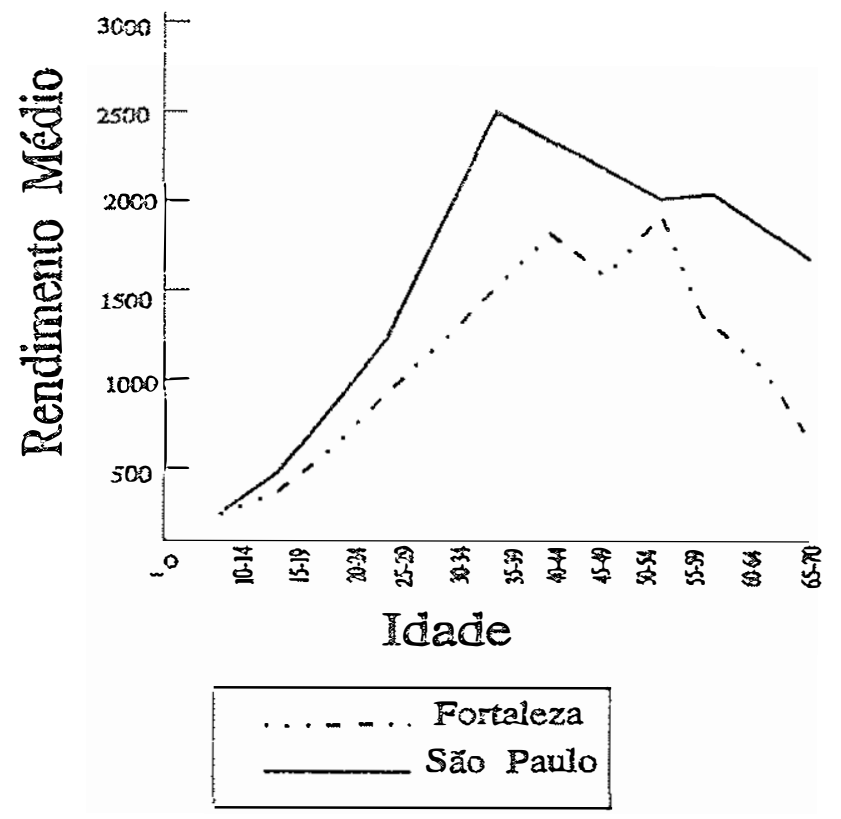

Figura 10.

Perfil etário de rendimentos médios

Fexmininos - a PEA 1985 


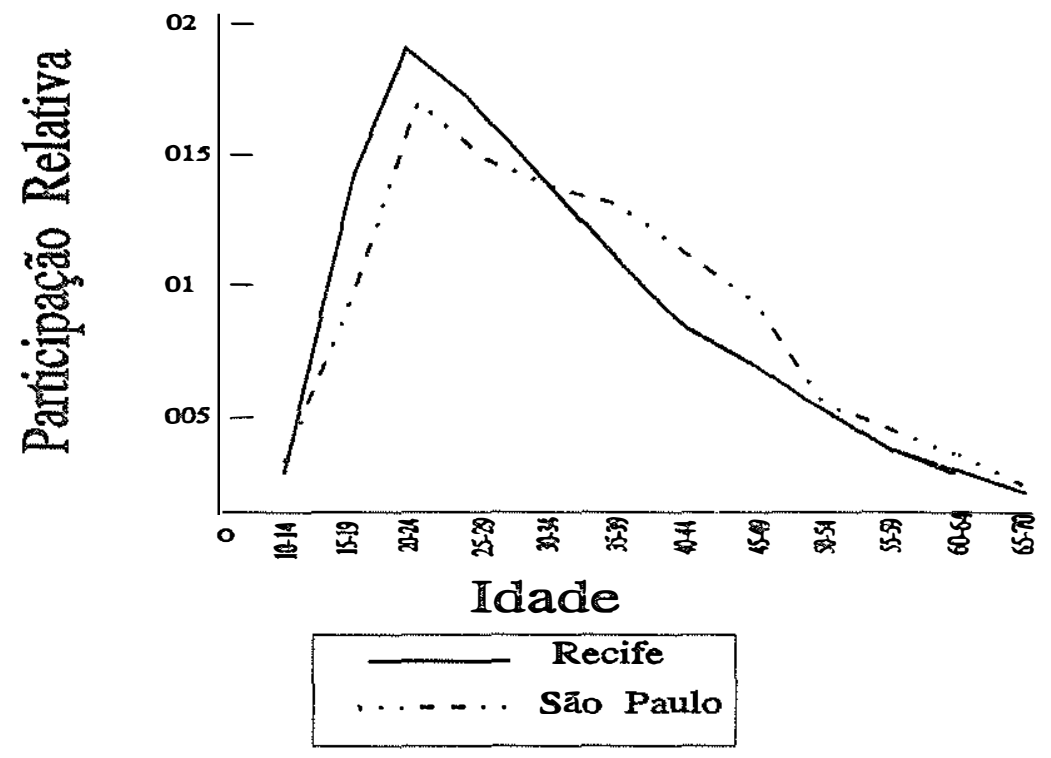

Figura 11.

Distribuição etária das PEAs

Feminina e masculina - São Paulo - 1985 


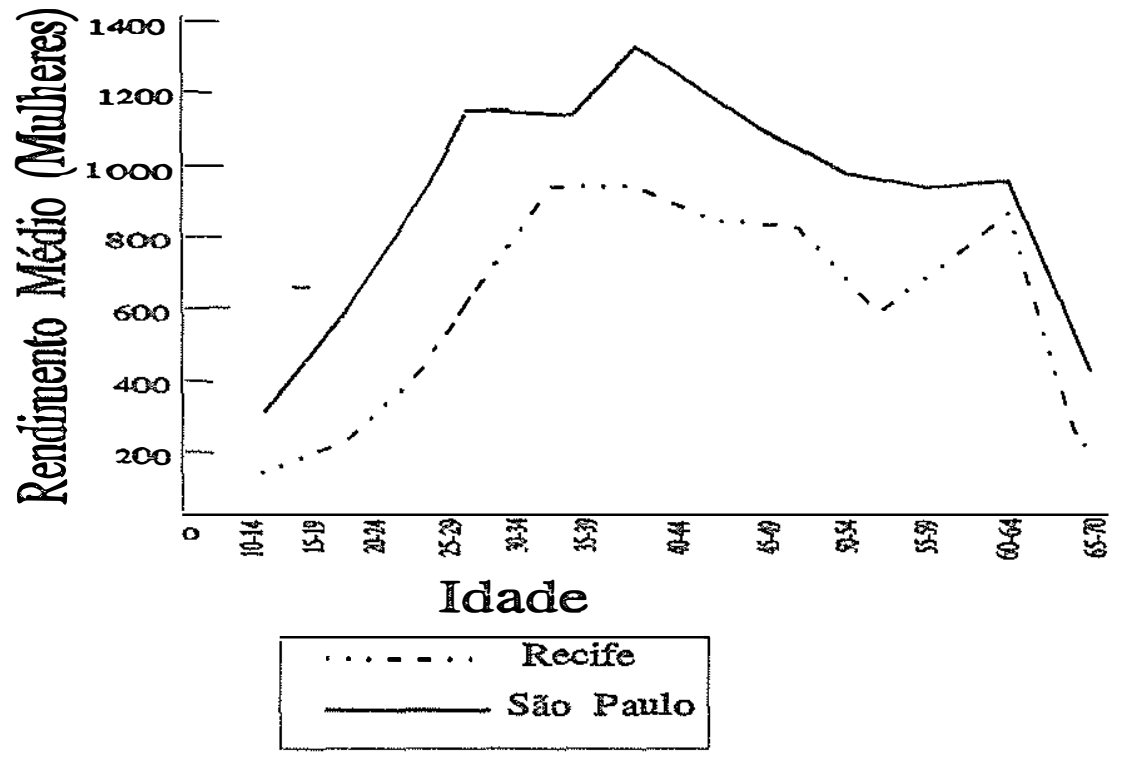

Figura 12.

Perfil etário das PEAs

Masculina e feminina - São Paulo - 1985 


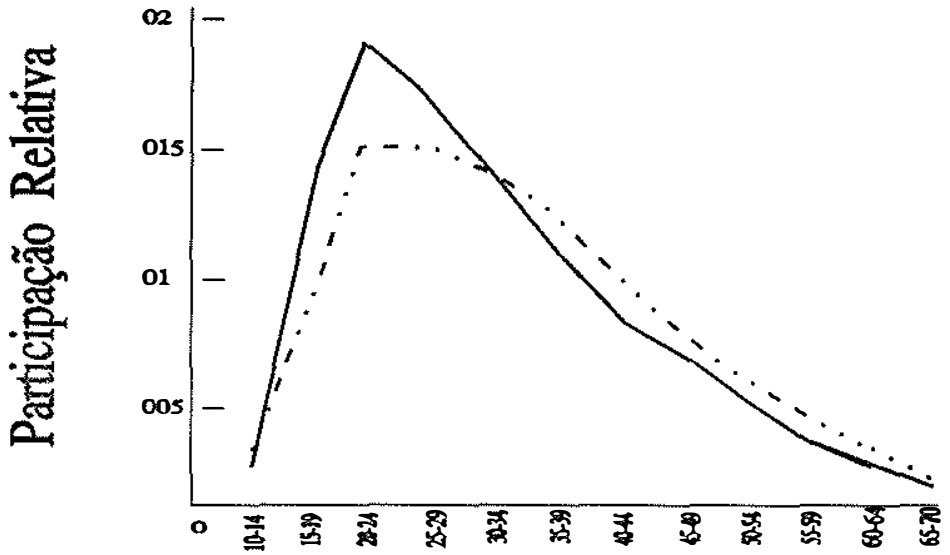

Idade

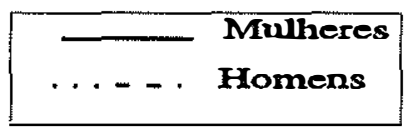

Figura 13.

Rendimento médio relativo

por regiōes metropolitanas - média 1976/1987 


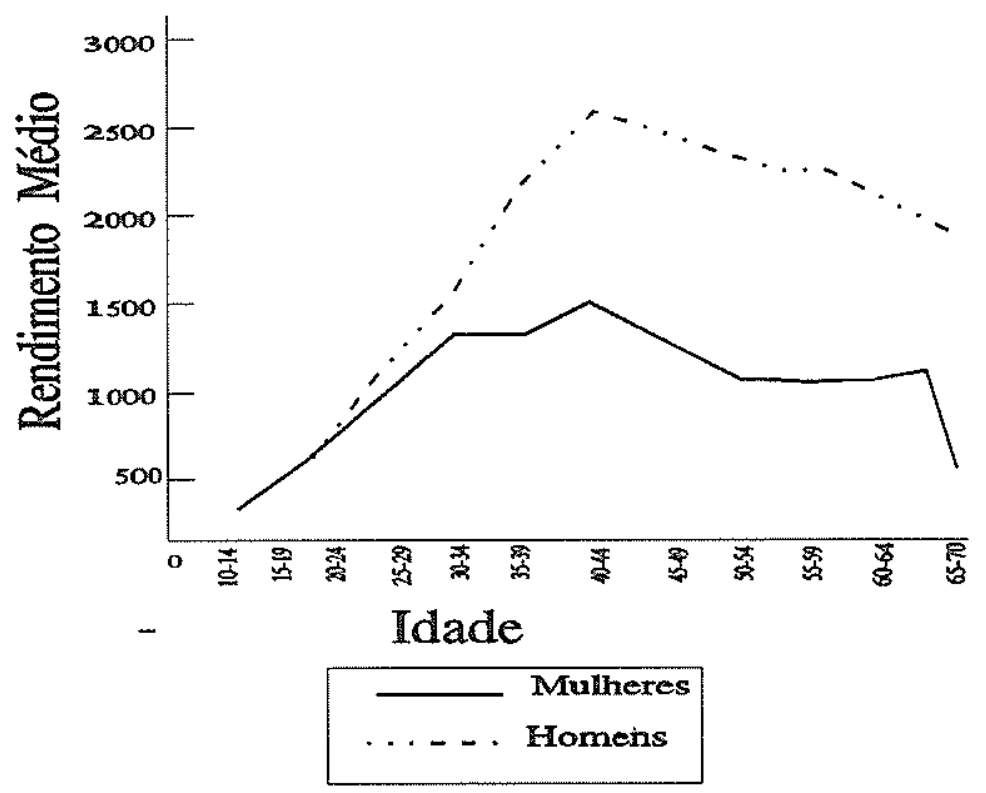

Figura 14.

Rendimento médio relativo

1976/1987 
